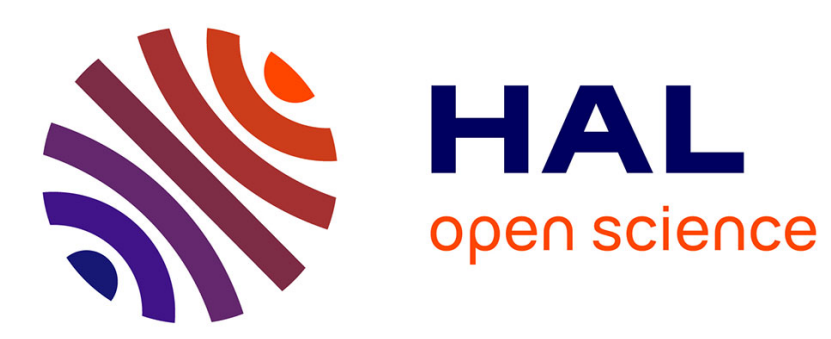

\title{
Multi-way Space-Time-WaveVector analysis for EEG source separation
}

Hanna Becker, Pierre Comon, Laurent Albera, Martin Haardt, Isabelle Merlet

\section{To cite this version:}

Hanna Becker, Pierre Comon, Laurent Albera, Martin Haardt, Isabelle Merlet. Multi-way SpaceTime-WaveVector analysis for EEG source separation. Signal Processing, 2012, 92 (4), pp.1021-1031. 10.1016/j.sigpro.2011.10.014 . hal-00683304

\section{HAL Id: hal-00683304 https://hal.science/hal-00683304}

Submitted on 28 Mar 2012

HAL is a multi-disciplinary open access archive for the deposit and dissemination of scientific research documents, whether they are published or not. The documents may come from teaching and research institutions in France or abroad, or from public or private research centers.
L'archive ouverte pluridisciplinaire HAL, est destinée au dépôt et à la diffusion de documents scientifiques de niveau recherche, publiés ou non, émanant des établissements d'enseignement et de recherche français ou étrangers, des laboratoires publics ou privés. 


\title{
Multi-way Space-Time-Wave-Vector analysis for EEG source separation is
}

\author{
Hanna Becker ${ }^{\mathrm{a}, \mathrm{b}, 1}$, Pierre Comon ${ }^{\mathrm{b}, 1}$, Laurent Albera ${ }^{\mathrm{c}, \mathrm{d}}$, Martin Haardt ${ }^{\mathrm{a}, 1, *}$, \\ Isabelle Merlet ${ }^{\mathrm{c}, \mathrm{d}}$ \\ ${ }^{a}$ Ilmenau University of Technology, Communications Research Laboratory, P. O. Box \\ 1005 65, D-98684 Ilmenau, Germany \\ ${ }^{b}$ Lab. I3S, UMR6070 CNRS, University of Nice, BP 121, F-06903 Sophia-Antipolis, \\ France \\ ${ }^{c}$ INSERM, U642, Rennes, F-35000, France \\ ${ }^{d}$ Universite de Rennes 1, LTSI, Rennes, F-35000, France
}

\begin{abstract}
For the source analysis of ElectroEncephaloGraphic (EEG) data, both equivalent dipole models and more realistic distributed source models are employed. Several authors have shown that the Canonical Polyadic Decomposition ( also called ParaFac) of Space-Time-Frequency (STF) data can be used to fit equivalent dipoles to the electric potential data. In this paper we propose a new multi-way approach based on Space-Time-Wave-Vector (STWV) data obtained by a 3D local Fourier transform over space accomplished on the measured data. This method can be seen as a preprocessing

\footnotetext{
${ }^{2}$ Parts of this paper have been published at the EURASIP European Signal Processing Conference (EUSIPCO 2010), Aalborg, Denmark, August 2010. This journal version of our EUSIPCO 2010 paper has been invited by Bjorn Ottersten, Soren Holdt Jensen, Bastiaan Kleijn and Jan Larsen (see attached manuscript notes).

*Corresponding author: e-mail: martin.haardt@tu-ilmenau.de, phone: +49 (3677) 692613, fax: +49 (3677) 69-1195

Email addresses: hanna.becker@tu-ilmenau.de (Hanna Becker), pcomon@unice.fr (Pierre Comon), laurent.albera@univ-rennes1.fr (Laurent Albera)

${ }^{1}$ Member of EURASIP
} 
step that separates the sources, reduces noise as well as interference and extracts the source time signals. The results can further be used to localize either equivalent dipoles or distributed sources increasing the performance of conventional source localization techniques like, for example, LORETA. Moreover, we propose a new, iterative source localization algorithm, called Binary Coefficient Matching Pursuit (BCMP), which is based on a realistic distributed source model. Computer simulations are used to examine the performance of the STWV analysis in comparison to the STF technique for equivalent dipole fitting and to evaluate the efficiency of the STWV approach in combination with LORETA and BCMP, which leads to better results in case of the considered distributed source scenarios.

Keywords: EEG, CanDecomp/ParaFac, Canonical Polyadic

decomposition, Source localization, LORETA,

Space-Time-Frequency/Space-Time-Wave-Vector analysis

\section{Introduction}

ElectroEncephaloGraphy (EEG) is a long-standing technique for the analysis of cerebral activity and consists in measuring the electric potential on the surface of the head with an array of sensors. Due to its good temporal resolution compared to other methods (like for example functional Magnetic Resonance Imaging (f-MRI)), the EEG is routinely used to record seizures in epileptic patients. An important issue is the identification of the epileptogenic zone, which can then be removed by surgery. To localize the sources based on the electric potential measured on the surface of the scalp, a multitude of different approaches has been proposed [1]. These methods vary 
mainly in the assumptions on the nature of the sources. In a first step, they can be distinguished into equivalent dipole models, which try to fit one dipole to each locally active cortical region, and distributed source models, which describe the neural activity everywhere in the brain with the help of thousands of dipolar sources.

One approach for the localization of equivalent dipoles is based on tensor decompositions applied to multi-way data. This technique requires a third dimension in addition to space and time. Several authors have studied the use of the Canonical Polyadic (CP) decomposition (also known as CanDecomp/ParaFac model) applied to Space-Time-Frequency (STF) data obtained by a wavelet transform over time $[2,3,4,5,6]$ or the application of the Wigner-Ville distribution [7]. The method was tested on simulated as well as real data and lead to promising results. But this technique depends on the source time signals, which are assumed to be oscillatory, and does not permit to separate several simultaneously active brain regions with correlated activities into more than one component, thus preventing the representation of such a scenario by an adequate number of equivalent dipole sources.

In this paper, a new CP based approach using a different dimension is explored. This technique is based on data transformed into the Space-TimeWave-Vector (STWV) domain and is obtained using a 3D local Fourier transform over space. The advantage of this method is that it does not depend on the temporal behavior of the sources. Instead, the STWV approach requires the sources to be superficial (i. e., at the surface of the cortex) in order to obtain meaningful results when applying the CP decomposition. However, since it is known that the activity recorded by EEG measurements is mostly 
generated by pyramidal cells located in the gray matter [8], this requirement is usually well met. Due to these properties, the STWV method permits to accurately localize one or several equivalent dipole sources and extract at the same time a good estimate of the source time signals. Contrary to the STF analysis, it also allows for the separation of correlated sources.

Furthermore, we propose to employ the results of the STWV method, which are characterized by an increased SNR compared to the original data, separated sources and already extracted source time signals, as a basis for distributed source localization procedures. To this end, we introduce a new distributed source localization algorithm, referred to as Binary Coefficient Matching Pursuit (BCMP). This algorithm is based on a distributed source model assuming radially oriented source dipoles of equal strengths that are located on the surface of the cortex. In [9], a similar model was shown to accurately explain measured electric potential data. As will be shown in the following, the BCMP algorithm outperforms the source localization results of the Low Resolution Electromagnetic Tomography (LORETA) technique [10], which is probably the best known distributed source localization method and has been reported to give more accurate results than other Weighted Minimum Norm algorithms [11]. LORETA selects the smoothest solution possible, assuming that adjacent neurons are synchronously active. However, this constraint also accounts for a relatively low spatial resolution producing blurred source localization results. Furthermore, it is noteworthy that standard LORETA does not supply a satisfactory estimate of the source time signals because the temporal information can only be considered by computing independent LORETA solutions for each time sample. On the contrary, 
the BCMP algorithm based on the STWV preprocessed data provides an accurate estimate of the source time signals.

The rest of the paper is organized as follows: Based on Section 2, which describes the EEG data model, we explain the concept of multilinear modeling for both the STF and the STWV analysis in Section 3. Subsequently, the source extraction and localization schemes for equivalent dipole fitting and distributed source modeling based on the preprocessed data are described in Section 4 and analyzed with the help of computer simulations in Section 5. A short summary of the results is given in Section 6 .

The following notation is used throughout this paper: bold italic uppercase letters denote tensors, e.g., $\boldsymbol{T}$, bold uppercase letters denote matrices, e.g., A, bold lowercase letters denote column vectors, e.g., a, and plain font denotes scalars, e.g., $X_{i j k}, T_{i j}$ or $a_{i}$. Moreover, $(\cdot)^{\mathrm{T}}$ denotes a transposition and $(\cdot)^{+}$stands for the Moore-Penrose pseudo-inverse.

\section{Data model}

The electric potential on the surface of the scalp can be recorded with an array of sensors as a function $x(\mathbf{r}, t)$ of electrode position $\mathbf{r}$ and time $t$. The obtained EEG data, which is sampled in space and time, can be stored in a data matrix $\mathbf{X} \in \mathbb{R}^{N_{r} \times N_{t}}$ where $N_{r}$ and $N_{t}$ denote the number of sensors and time samples, respectively. Assuming a static propagation medium, this matrix can be factorized into a leadfield matrix $\mathbf{A}^{(0)} \in \mathbb{R}^{N_{r} \times R}$, depending on spatial parameters (geometry and conductive properties of the head) and a signal matrix $\mathbf{S} \in \mathbb{R}^{R \times N_{t}}$, which describes the temporal variations of the $R$ current sources: 


$$
\mathbf{X}=\mathbf{A}^{(0)} \mathbf{S}
$$

In practice, the measurements are subject to noise which leads to the perturbed data matrix

$$
\tilde{\mathbf{X}}=\mathbf{A}^{(0)} \mathbf{S}+\mathbf{N}
$$

where $\mathbf{N}$ is the noise matrix. In this paper, we assume that the noise is generated by noise dipoles located on the surface of the cortex not covered by the source dipoles, which emit a zero-mean white Gaussian background activity. This leads to temporally white, but spatially correlated additive noise on the scalp. In practice, EEG measurements are also subject to artifacts, e.g., due to eye movements and muscle activity. In this paper, we assume that artifacts and non-Gaussian EEG rhytms that are not of interest have been removed prior to the data analysis.

The description of the leadfield matrix $\mathbf{A}^{(0)}=\left[\mathbf{a}_{1}^{(0)} \ldots \mathbf{a}_{R}^{(0)}\right]$ depends on both the source model and the used head model. In case of the equivalent dipole model, each of the leadfield vectors $\mathbf{a}_{r}^{(0)}, r=1 \ldots R$, is a function of the position and orientation of one equivalent dipole.

For the development of the BCMP algorithm, we introduce the following definition and model of a distributed source: According to [9], a distributed source can be described as the union of (one or) several non-necessarily contiguous areas of the cortex (so-called patches) with highly correlated source activities. This source region can be represented by a number of dipolar sources each of which models thousands of simultaneously active neurons that are arranged in parallel and oriented perpendicular to the surface of the cortex. The distributed source model thus assumes the following:

1. The underlying dipolar sources are located exclusively on the surface 
of the cortex.

2. The dipole sources are radially oriented.

Additionally, we assume that

3. all dipole sources of a given distributed source have the same strength.

The leadfield vector $\mathbf{a}^{(0)}$ of one distributed source can then be described as a superposition of the leadfield vectors $\mathbf{d}\left(\boldsymbol{\rho}_{k}\right)$ of $K$ source dipoles where $\boldsymbol{\rho}_{k}$ is the position of the $k$-th dipole source:

$$
\mathbf{a}^{(0)}=\sum_{k=1}^{K} \mathbf{d}\left(\boldsymbol{\rho}_{k}\right)
$$

The computation of the leadfield vectors for given dipole parameters is performed either analytically or numerically, depending on the head model employed [12].

\section{Multilinear modeling}

The data model (1) is a bilinear model in space and time. The goal is to recover the leadfield and signal matrices by separating the measured data into spatial and temporal characteristics. However, for a matrix decomposition to be unique, constraints like, for example, orthogonality or statistical independence can be imposed, as incorporated in Principal Component Analysis (PCA) or Independent Component Analysis (ICA), respectively [13]. Since there is no physiological justification for such constraints, especially in epilepsy where different distributed sources can be partially correlated, another solution to the problem of non-uniqueness is desirable. This is where 
the CP decomposition comes into play. The CP model can be used to approximate a tensor $\boldsymbol{X}$ of size $I \times J \times K$ and rank $Q$ by a tensor $\hat{\boldsymbol{X}}$ of given lower rank $P$ :

$$
\hat{\boldsymbol{X}}=\sum_{p=1}^{P} \boldsymbol{T}(p),
$$

$\boldsymbol{T}(p)$ being decomposable tensors of the form

$$
T_{i j k}(p)=\gamma_{p} \cdot a_{i}(p) b_{j}(p) c_{k}(p)
$$

where $\gamma_{p}$ denote the CP component amplitudes and $a_{i}(p), b_{j}(p)$ and $c_{k}(p)$ are stored in three loading matrices $\mathbf{A}, \mathbf{B}$ and $\mathbf{C}$ of sizes $I \times P, J \times P$ and $K \times P$, respectively, with unit norm columns; see [14] [15] and references therein. Equation (4) is referred to as the CP model and comprises a trilinear structure. The crucial point is that one may consider that there are almost surely finitely many $\mathrm{CP}$ decompositions (for $\hat{\boldsymbol{X}}$ ) if $P<\frac{I J K}{I+J+K-2}$, even if to date, this conjecture is fully proved only in the symmetric case. On the other hand, Kruskal's lemma provides non necessary but sufficient conditions for a unique CP decomposition to exist; see [15] and references therein.

It is worth noting that, even when the CP (4) is unique, its representation (5) with three loading matrices will always be subject to (the same) permutation of their columns, which are themselves identified up to a multiplicative scalar factor of unit modulus. This indeterminacy is inherent in the representation of decomposable tensors, and has nothing to do with the uniqueness of decomposition (4). 


\subsection{Space-Time-Frequency (STF) analysis}

To apply the CP tensor model to EEG data, a transformation needs to be found which turns the data matrix into a data tensor. One possibility to collect a third diversity consists in computing the wavelet transform (or a short term Fourier transform) over time of the measured electric potential data $[2,3,4,5,6]$. The resulting tensor $\boldsymbol{W}$ depends on space $\mathbf{r}$, time $t$, and frequency $f$ :

$$
W(\mathbf{r}, t, f)=\int_{-\infty}^{\infty} x(\mathbf{r}, \tau) \psi(a, \tau, t) \mathrm{d} \tau
$$

The frequency $f$ can be estimated from the scale $a$ of the wavelet $\psi(a, \tau, t)$ by $f=f_{c} /(a \cdot T)$ where $f_{c}$ is the center frequency of the wavelet and $T$ is the interval between time samples.

If the frequency content of each of the EEG source signals can be assumed to be time-invariant except for a scaling factor, which is the case for oscillatory signals, the time and frequency variables separate. Thus, the tensor $\boldsymbol{W}$ approximately comprises a trilinear structure and can be decomposed using the CP model (4-5):

$$
W\left(\mathbf{r}_{i}, t_{j}, f_{k}\right) \approx \sum_{p=1}^{P} \gamma_{p} \mathbf{a}\left(\mathbf{r}_{i} ; p\right) \mathbf{b}\left(t_{j} ; p\right) \mathbf{c}\left(f_{k} ; p\right)
$$

Here, $\mathbf{r}_{i}, t_{j}$ and $f_{k}$ represent the sampled space, time and frequency variables and $\mathbf{a}\left(\mathbf{r}_{i} ; p\right), \mathbf{b}\left(t_{j} ; p\right)$ and $\mathbf{c}\left(f_{k} ; p\right)$ denote elements of the loading matrices $\mathbf{A}, \mathbf{B}$ and $\mathbf{C}$ of the $\mathrm{CP}$ model indicating the space, time and frequency characteristics, respectively. In the absence of noise, for decorrelated sources, the number of dominant components $P$ of the $\mathrm{CP}$ model equals the number of sources $R$, whereas $P$ generally equals $R+1$ in a noisy environment $[2,3]$ because noise accounts for an additional component in (7). 


\subsection{Space-Time-Wave-Vector (STWV) analysis}

The new idea of this paper consists in applying a transformation to the space variable instead of the time variable. If a 3D local Fourier transform of the electric potential data is computed over space, a third order tensor $\boldsymbol{F}$ is obtained where the third variable is now the wave vector $\mathbf{k}$.

$$
F(\mathbf{r}, t, \mathbf{k})=\int_{-\infty}^{\infty} w\left(\mathbf{r}^{\prime}-\mathbf{r}\right) x\left(\mathbf{r}^{\prime}, t\right) e^{j \mathbf{k}^{\mathrm{T}} \mathbf{r}^{\prime}} \mathrm{d} \mathbf{r}^{\prime}
$$

Here, $w\left(\mathbf{r}^{\prime}-\mathbf{r}\right)$ denotes a $3 \mathrm{D}$ window function centered at $\mathbf{r}$ which selects electric potential data within a certain region of the scalp to be used for the Fourier transform at point $\mathbf{r}$.

Similarly to the STF approach, a CP decomposition of the sampled tensor $\boldsymbol{F}$ given by

$$
F\left(\mathbf{r}_{i}, t_{j}, \mathbf{k}_{l}\right) \approx \sum_{p=1}^{P} \gamma_{p} \mathbf{a}\left(\mathbf{r}_{i} ; p\right) \mathbf{b}\left(t_{j} ; p\right) \mathbf{c}\left(\mathbf{k}_{l} ; p\right)
$$

into a finite number of dominant components $P$ that equals the number of sources is possible if the data fulfills the following property: For each source the spatial frequency content of the leadfield vector $\mathbf{a}^{(0)}$ ( including the directions of the associated dominant wave vectors ) has to be the same at every sensor, except for a scaling factor. However, in practice, the dominant wave vectors, which indicate the direction of the largest changes of electric potential, can differ a lot at two distant sensors, thus violating the requirement for a trilinear model. This problem can be overcome by the fact that superficial sources as examined in this paper only produce great potential values within small regions (see Figures 2 (top right) and 1 for an example of the electric potential distribution and the space-wave-vector data generated by a dipole source at one time instant). At adjacent sensors the spatial frequency content associated to the dominant wave vectors can then be assumed to be 
approximately the same up to scaling factors, and the differences at distant sensors can be neglected.

Figure 2 shows an example of the space, time and wave vector characteristics obtained after the decomposition of the tensor $\boldsymbol{F}$ of a dipole source.

\section{Source extraction and localization}

After separating the EEG data into several components associated with different sources using the CP model, the source time signals can be extracted and the source positions can be estimated. Whereas the source extraction can be achieved by simply estimating the signal matrix $\mathbf{S}$, the source localization concept consists of two steps:

1. Compute an estimate of the leadfield matrix $\mathbf{A}^{(0)}$.

2. Determine the source parameters (dipole positions and orientation, if not assumed to be radial).

The estimation of the signal matrix and the leadfield matrix will be described in detail for both STF and STWV decompositions in the context of equivalent dipoles. Once these estimates are obtained, the preprocessing of the EEG data is completed and the actual source localization can follow.

This procedure has several advantages over source localization on the raw data which are due to the previous signal processing steps (tensor decomposition and leadfield as well as signal matrix estimation):

- The electric potential distribution has been separated into components each of which corresponds to only one distributed source. The source localization process can thus be applied to each source separately. 
- The noise has been reduced.

- The source time signals have already been extracted, which is, according to [1], for example not satisfactorily achieved with LORETA.

\subsection{Equivalent dipole fitting}

\subsubsection{Source extraction}

Due to the different properties of the STF and STWV models, the methods used for the estimation of the signal matrix differ (cf. Figure 3). In the case of the STF analysis, an exact separation of the Wavelet transformed data into time and frequency characteristics can only be obtained if the frequency content of the signal is constant over time (cf. Section 3.1). In practice, this is not the case and the bilinear approximation of the time-frequency data limits the accuracy of the time signals estimated by the temporal characteristics. This is why we use the pseudo-inverse of the estimated leadfield matrix $\hat{\mathbf{A}}^{(0)}$ (see Section 4.1.2) to obtain an improved estimate of the signal matrix $\hat{\mathbf{S}}$ in equation (1). This can be problematic if $\hat{\mathbf{A}}^{(0)}$ is not a tall matrix, meaning that there are more sources than sensors. However, in the case of equivalent dipole models, the number of sought sources usually does not exceed 3 or 4 , which is much smaller than the number of electrodes.

By contrast, the temporal characteristics extracted by the CP decomposition of the STWV tensor $\boldsymbol{F}$ already constitute an accurate approximation $\hat{\mathbf{S}}$ of the signal matrix $\mathbf{S}$. This property is due to the fact that the Fourier transform over space does not affect the source activities, which means that the elements $F\left(\mathbf{r}_{i}, t_{j}, \mathbf{k}_{l}\right)$ of the tensor $\boldsymbol{F}$ admit the exact bilinear model

$$
F\left(\mathbf{r}_{i}, t_{j}, \mathbf{k}_{l}\right)=\sum_{r=1}^{R} b\left(t_{j} ; r\right) D\left(\mathbf{r}_{i}, \mathbf{k}_{l} ; r\right)
$$


where $b\left(t_{j} ; r\right)$ is the activity of source $r$ at time $t_{j}$.

\subsubsection{Source localization}

In the case of the STF method the Wavelet transform over time of the electric potential data does not affect the leadfield matrix, which means that the spatial characteristics on the one side and the combined time-frequency characteristics on the other side still form a perfect bilinear model. Therefore, the loading matrix $\mathbf{A}$ containing the spatial characteristics extracted with the STF approach constitutes a good approximation for the leadfield matrix $\mathbf{A}^{(0)}$.

However, the 3D local Fourier transform over space accomplished to obtain the tensor $\boldsymbol{F}$ of the STWV approach does not lead to a bilinear model with clearly separated space and wave vector characteristics. Consequently, the loading matrix A of the STWV method does not permit to accurately localize the dipole sources, and another approach based on the signal matrix has to be taken (cf. Figure 3). Once an accurate estimate $\hat{\mathbf{S}}$ of the signal matrix is available (see Section 4.1.1), the leadfield matrix can be computed from:

$$
\hat{\mathbf{A}}^{(0)}=\mathbf{X} \hat{\mathbf{S}}^{+}
$$

using the original data. Note that, since it is always possible to have more time samples than sources, the computation of the pseudo-inverse $\hat{\mathbf{S}}^{+}$of the signal matrix $\hat{\mathbf{S}}$ does not raise any problem.

Once the leadfield estimation has been accomplished, the source parameters, namely location and orientation of the equivalent dipoles which best match the estimated leadfield in a least squares sense need to be determined. To this end, a non-linear least squares algorithm can be employed provided that an analytical expression for the leadfield vectors is available. 


\subsection{Distributed source modeling}

As a distributed source can comprise an area of up to $20 \mathrm{~cm}^{2}$ of simultaneously activated brain tissue, a separation into several CP components can occur. This is due to the fact that the spatial Fourier transform is computed for smaller patches. The active region of the cortex is then characterized by the space-wave-vector data of several sensors which can be decomposed into different components. These components have to be identified as belonging to the same distributed source. To this end, one can exploit the fact that the neuronal activity within a distributed source is highly correlated. Thus, the association of several CP components to one distributed source is characterized by a correlation coefficient between their temporal characteristics which exceeds a certain threshold. To obtain a representative signal vector for each distributed source, the signal vectors associated with the $\mathrm{CP}$ components belonging to this source are then simply added. Analogously, an estimate of the leadfield matrix can be determined by summing up all the leadfield vectors of the $\mathrm{CP}$ components which belong to the same distributed sources. Please note that to obtain meaningful results, it is important to include the component amplitudes in the CP model. In case of the STF analysis, they have to be considered in the spatial characteristics whereas for the STWV approach, they need to be incorporated in the temporal characteristics.

The objective of distributed source localization then consists in providing a better estimate of the actual spatial form of the distributed sources, based on the leadfield vector associated with each of the sources. 


\subsubsection{Cortical LORETA}

In fact, mathematically, the source localization based on the leadfield vectors extracted from the data matrix with the help of the CP decomposition leads to the same problem as other distributed source localization techniques try to solve, i.e., finding a solution $\mathbf{c}$ to

$$
\hat{\mathbf{a}}^{(0)}(r)=\mathbf{D} \cdot \mathbf{c}
$$

Here, $\hat{\mathbf{a}}^{(0)}(r), r=1 \ldots R$, is the estimated leadfield vector of the $r$-th source,

the matrix $\mathbf{D}=\left[\mathbf{d}_{1} \ldots \mathbf{d}_{N_{d}}\right] \in \mathbb{R}^{N_{r} \times N_{d}}$ contains the leadfield vectors of all grid dipoles and the solution vector $\mathbf{c}$ contains a set of continuous real-valued coefficients that can be interpreted as the strengths of the grid dipoles. In order to find a unique solution to this underdetermined set of equations, various constraints can be imposed. Thus, an algorithm like, for example, Low Resolution Electromagnetic Tomography (LORETA), which searches for the smoothest solution to equation (12), can be applied to the preprocessed data. In order to incorporate the physiology-based assumptions of superficial, radially oriented sources, the original LORETA algorithm [10] can be modified in the following way:

1. Rather than employing a 3D grid that permeates the whole head, a grid that covers only the surface of the cortex is used.

2. Instead of looking for the 3 components describing the dipole moment vector and dipole strength at each vertex of the grid, only the dipole strength is determined since its orientation is assumed to be radial.

This modified version of LORETA is subsequently referred to as cortical LORETA. 
As the inverse problem that is solved in case of the original LORETA algorithm is mathematically the same as equation (12), we can use its explicit solution given in [10]. Thus, the vector

$$
\mathbf{c}_{l o r}=\left(\mathbf{O L}^{\mathrm{T}} \mathbf{L O}\right)^{-1}(\mathbf{D})^{\mathrm{T}}\left[\mathbf{D}\left(\mathbf{O L}^{\mathrm{T}} \mathbf{L O}\right)^{-1}(\mathbf{D})^{\mathrm{T}}\right]^{+} \hat{\mathbf{a}}^{(0)}
$$

contains the cortical LORETA solution for the strength of each of the grid dipoles, where $\mathbf{O}$ is a diagonal matrix with $O_{i, i}=\sqrt{\left(\mathbf{d}_{i}\right)^{\mathrm{T}} \mathbf{d}_{i}}, i=1 \ldots N_{d}$ and L implements the spatial Laplacian operator [10] (see [16] for an implementation based on a non-uniform grid), ensuring that the LORETA constraint of the smoothest possible solution is fulfilled. Choosing a certain threshold value for these dipole amplitudes, all the dipoles whose strength exceeds the threshold can be said to belong to the distributed source.

\subsubsection{Binary Coefficient Matching Pursuit (BCMP)}

Since the spatial resolution of the results that LORETA supplies is relatively low and this method is not well suited for the localization of focal sources, we propose another distributed source localization scheme which is based on the distributed source model described above.

The objective of this algorithm consists in identifying the grid dipoles which lead to the best approximation of the estimated leadfield by solving the following combinatorial optimization problem:

$$
\min _{\mathbf{i}}\left\|\hat{\mathbf{a}}^{(0)}(r)-m \cdot \mathbf{D} \cdot \mathbf{i}\right\|^{2}
$$

where $\hat{\mathbf{a}}^{(0)}(r), r=1 \ldots R$, is the estimated leadfield vector of the $r$-th source (cf. equation (3)), $m$ is a normalization factor due to the scaling ambiguity of the CP model, $\mathbf{D}$ contains the leadfield vectors of all grid dipoles and $\mathbf{i}$ is a coefficient vector whose elements are binary (either 1 or 0 ). As most of 
the coefficients of the vector $\mathbf{i}$ in equation (14) are 0 , this corresponds to a sparse representation of the distributed source from grid dipoles. This fact has motivated the following approach, which is based on the Matching Pursuit algorithm [17]. The main difference consists in the restriction to binary coefficients in the present problem. The resulting Binary Coefficient Matching Pursuit (BCMP) algorithm comprises the following steps and should be executed for every source leadfield vector:

1. Initialization: Set the coefficient vector $\mathbf{i}=\mathbf{0}_{N_{d}, 1}$, where $\mathbf{0}_{N_{d}, 1}$ is a vector of size $N_{d} \times 1$ whose elements are 0 , and the number of iterations $k=1$. Find the vector $\mathbf{d}_{i}$ of $\mathbf{D}$ which leads to the minimal metric. The dictionary $\mathbf{D}^{(1)}$ is obtained by removing the vector $\mathbf{d}_{i}$ from $\mathbf{D}$.

Set $\hat{\mathbf{a}}^{(1)}=\mathbf{d}_{i}, \mathbf{i}^{(1)}(i)=1$ and the metric at iteration $1 \operatorname{met}(1)=$ $\left\|\hat{\mathbf{a}}^{(0)}(r)-m^{(1)} \cdot \hat{\mathbf{a}}^{(1)}\right\|^{2}$, where

$$
m^{(k)}=\frac{\sum_{n=1}^{N_{r}}\left|\hat{a}_{n}^{(k)}\right|}{\sum_{n=1}^{N_{r}}\left|\hat{a}_{n}^{(0)}(r)\right|}
$$

is the normalization factor at iteration $k$ which needs to be introduced due to the scaling indeterminacy of the decomposition.

2. At the $(k+1)$-th step, set $\mathbf{i}^{(k+1)}=\mathbf{i}^{(k)}$, find the vector $\mathbf{d}_{j}$ of $\mathbf{D}^{(k)}$ which minimizes

$$
\operatorname{met}(k+1)=\min _{j}\left\|\hat{\mathbf{a}}^{(0)}(r)-m^{(k+1)} \cdot\left(\hat{\mathbf{a}}^{(k)}+\mathbf{d}_{j}\right)\right\|^{2}
$$

and update the estimated leadfield: $\hat{\mathbf{a}}^{(k+1)}=\hat{\mathbf{a}}^{(k)}+\mathbf{d}_{j}$.

Find the index $i$ of the leadfield vector $\mathbf{d}_{i}$ in $\mathbf{D}$ corresponding to the leadfield vector $d_{j}$ and set $\mathbf{i}^{(k+1)}(i)=1$. Remove vector $\mathbf{d}_{j}$ from the dictionary $\mathbf{D}^{(k)}$ to obtain $\mathbf{D}^{(k+1)}$. Increment the iteration index: $k=$ $k+1$. 
3. Stop after a given number of iterations $k_{\max }$ is reached and return the index vector $\mathbf{i}^{\left(k_{\max }\right)}$. This vector indicates the indices of the grid dipoles whose leadfield vectors are stored in the original dictionary $\mathbf{D}$ and which are thus associated with the distributed source.

To ensure a physiologically reasonable result, small holes in the form of the distributed source can be filled and isolated grid dipoles associated to the distributed source, which occur in the case of noise, can be removed by applying a smoothing procedure.

The algorithm can be accelerated remarkably by considering only dipoles of the cortical grid which are located under a scalp region with high electric potential values, corresponding to the expanded distributed source region.

\section{Simulation results}

To examine the performance of the STWV analysis for source extraction and localization, computer simulations are performed. In the context of equivalent dipoles, the STWV analysis is compared to the STF technique. Furthermore, following the STWV analysis, we compare the distributed source localization results obtained with the BCMP algorithm to those of cortical LORETA.

In general, the simulation consists of the following steps: For a given source configuration of radially oriented dipoles, the leadfield is computed with the help of a 3 -shell spherical head model. The radii of the 3 shells representing brain, skull and scalp are $8 \mathrm{~cm}, 8.5 \mathrm{~cm}$ and $9.2 \mathrm{~cm}$ and their conductivities are set to $3.3 \times 10^{-3} \mathrm{~S} / \mathrm{cm}, 8.25 \times 10^{-5} \mathrm{~S} / \mathrm{cm}$ and $3.3 \times 10^{-3}$ $\mathrm{S} / \mathrm{cm}$, respectively. Epileptiform signals are generated with the help of the 
Jansen model [18] for a sampling interval of $T=0.008 \mathrm{~s}$.

A noise matrix containing temporally white, but spatially correlated noise is computed in the same way as the data matrix for given noise dipoles with radial orientation emitting white Gaussian signals. The noise sources are chosen from a cortical grid such that they do not correspond to equivalent dipoles and do not belong to distributed sources. To produce an approximately uniform grid on the inner sphere with $5 \mathrm{~mm}$ distance between adjacent dipoles, a model referred to as cubed sphere is used. The noise matrix is normalized to match a given $\operatorname{SNR} P_{s} / P_{n}$, where $P_{n}$ is the power of the noise and $P_{s}=\frac{1}{N_{r} \cdot N_{t}} \sum_{i=1}^{N_{r}} \sum_{j=1}^{N_{t}} \mathbf{X}_{i, j}^{2}$ is the signal power. Then the noise matrix is added to the data matrix according to (2).

Then the data tensor $\boldsymbol{F} \in \mathbb{C}^{N_{r}^{\prime} \times N_{t} \times N_{k}}$ is constructed for $N_{k}=63$ fixed wave vector samples according to the STWV approach by computing a 3D non-uniform discrete local Fourier transform over space of the noisy electric potential data. To ensure meaningful results, the transform is only computed for $N_{r}^{\prime}$ sensors that are surrounded by at least 9 other electrodes within a certain distance, selected by employing the following spherical Blackman window function

$$
w\left(\mathbf{r}^{\prime}-\mathbf{r}\right)=0.42+0.5 \cdot \cos \left(2 \pi \frac{\left\|\mathbf{r}^{\prime}-\mathbf{r}\right\|}{\Delta r}\right)+0.08 \cdot \cos \left(4 \pi \frac{\left\|\mathbf{r}^{\prime}-\mathbf{r}\right\|}{\Delta r}\right)
$$

centered at point $\mathbf{r}$ (compare Figure 4). The diameter $\Delta r$ of the window function is adapted according to each sensor configuration, such that the data of sensors at the boundary of the array is not transformed. The wave vector samples $\mathbf{k}=\left[k_{1}, k_{2}, k_{3}\right]^{\mathrm{T}}$ are arbitrarily chosen to contain all possible combinations of $k_{1}, k_{2}, k_{3} \in\{0, \pm 1, \pm 2\}$ such that there are no two wave vectors $\mathbf{k}_{1}$ and $\mathbf{k}_{2}$ for which $\mathbf{k}_{1}=-\mathbf{k}_{2}$. 
Subsequently, the tensor is decomposed with the help of a semi-algebraic CP algorithm which is based on joint matrix diagonalizations [19], followed by one step of an Alternating Least Squares algorithm to ensure a realvalued signal matrix. The rank used for the decomposition is determined with the Corcondia algorithm [20]. For equivalent dipoles, the rank of $\boldsymbol{F}$ corresponds to the number of sources whereas the CP components still have to be regrouped in the case of distributed sources. If the correlation coefficient between the temporal characteristics of two CP components exceeds a threshold of $80 \%$, the components are assumed to belong to the same distributed source. The next step after the CP decomposition consists in the estimation of the leadfield and signal matrices as described in Section 3.2 for the STWV analysis. The results can then be used for source localization.

\subsection{Equivalent dipole fitting}

In this section, EEG data generated from one radially oriented, superficial equivalent dipole is analyzed. The data is recorded by 64 electrodes in order to obtain sufficient information for the computation of the spatial Fourier transform. To compare the results of the STWV analysis to those obtained with the STF analysis, we additionally compute a discrete Wavelet transform of the electric potential data using a real-valued Morlet-Wavelet. The resulting tensor $\boldsymbol{W} \in \mathbb{R}^{N_{r} \times N_{t} \times N_{f}}$ where $N_{f}=N_{t}$ stands for the number of frequency samples is equally decomposed and signal and leadfield matrices are estimated according to the STF analysis described in Section 3.1. Eventually, the positions of the equivalent dipoles are estimated by a non-linear least squares algorithm for both the STWV and the STF analysis. Then the Source Localization Errors (SLE) are computed over $N$ realizations according 
to

$$
\mathrm{SLE}=\frac{1}{N} \sum_{n=1}^{N} \sqrt{\left\|\hat{\boldsymbol{\rho}}_{n}-\boldsymbol{\rho}_{n}\right\|^{2}}
$$

where $\hat{\boldsymbol{\rho}}_{n}$ and $\boldsymbol{\rho}_{n}$ denote the estimated and the original positions of the $n$-th realization, respectively. Moreover, to evaluate the source extraction results, the correlation coefficient between the original and the estimated source time signals is calculated for both methods.

Number of time samples. To examine the influence of the number of time samples on the performance of the multi-way methods, the correlation coefficient between the estimated and the original signals, and the RMSE localization error of a source located at $\boldsymbol{\rho}=[-\pi / 12, \pi / 5,8 \mathrm{~cm}]$ (in spherical coordinates) are determined for different numbers of time samples for simulated EEG data recorded with 64 electrodes at a SNR of -3 dB; see Figure 5. The results consist of the outcome of 1000 trials and show that the STWV method still permits to localize the dipole source if only very short time samples are used whereas at least 150 time samples are necessary for the STF analysis to give as accurate results. If the tensors of both approaches are of the same size (which is the case for $N_{k}=63$ time samples), the STWV method clearly leads to better results for both source localization and extraction.

Influence of noise. Since EEG data is usually very noisy, an important issue of source localization methods is their robustness to noise. In the following simulation for a sensor array composed of 64 electrodes, the influence of spatially correlated, temporally white Gaussian noise on the source localization accuracy is examined for both STWV and STF analyses in case of a dipole source positioned at $\boldsymbol{\rho}=[\pi / 2, \pi / 8,8 \mathrm{~cm}]$ (in spherical coordinates) and 100 
time samples. Subsequent results, displayed in Figure 6, constitute an average over at least 100 trials with different noise and signal matrices. For a SNR of $-4 \mathrm{~dB}$ or better, the source localization error of the STWV analysis drops below $1 \mathrm{~cm}$ and the correlation coefficient exceeds $90 \%$, which means that the source parameters are well estimated. On the contrary, for the STF approach to yield as accurate results, the SNR needs to be at least $0 \mathrm{~dB}$. Thus the STWV method is clearly more robust to noise than the STF analysis. This can be explained by the fact that the STWV technique reduces the temporally white noise by averaging over time when the leadfield matrix is calculated from the pseudo-inverse of the estimated signal matrix $\hat{\mathbf{S}}$. Hence the noise on the STWV leadfield matrix is diminished.

On the contrary, the STF method tries to eliminate the noise on the signal matrix by averaging over space, which does not eliminate the spatially correlated noise. Moreover, the STF analysis partly separates the noise into an additional component of the CP model, which is often not as efficient for denoising as the procedure of the new STWV analysis.

\subsection{Distributed source modeling}

A distributed source comprises several circular-shaped patches defined by the center of the patch and its area. It is described by dipoles chosen from a cortical grid. The epileptiform signal of each distributed source is superimposed with white Gaussian noise to create highly correlated activities with a correlation coefficient of about $95 \%$ for all source dipoles. For all distributed source simulations, data is generated for 128 electrodes, 100 time samples and a SNR of $-3 \mathrm{~dB}$ if not stated otherwise.

After preprocessing the data with the help of the STWV analysis, the 
distributed sources are localized using the BCMP algorithm, which is followed by a smoothing operation (described in subsection 4.2.2).

For comparison, a cortical LORETA solution to the source localization problem is computed. Here, two cases are examined:

1. Cortical LORETA is run on the raw EEG data, averaged over all time samples.

2. Cortical LORETA is applied to the preprocessed data following the proposed STWV analysis.

For both the BCMP algorithm and cortical LORETA, the number of dipoles sought is fixed to approximately match the number of grid dipoles of the original distributed source ${ }^{2}$.

To evaluate the results, a measure called the True Positive Fraction (TPF), which contains the percentage of correctly identified distributed source dipoles, is employed. The TPF is defined by

$$
\mathrm{TPF}=\frac{\#\{\mathcal{I} \cap \hat{\mathcal{I}}\}}{\#\{\mathcal{I}\}}
$$

where $\mathcal{I}$ and $\hat{\mathcal{I}}$ denote the indices in the sets of grid dipoles forming the original distributed source and the estimated distributed source, respectively, and $\#\{\mathcal{I}\}$ denotes the number of elements in the set $\mathcal{I}$.

Number of time samples. As for the equivalent dipole simulations, the objective of the first simulation is to examine the influence of the number of time samples. To this end, a distributed source comprising an area of approximately $16 \mathrm{~cm}^{2}$ and composed of three circular-shaped patches of sizes $5 \mathrm{~cm}^{2}$,

\footnotetext{
${ }^{2}$ In practice, the number of dipoles could, for example, be determined by searching for the minimal metric met $(k)$ that occurs during the BCMP algorithm.
} 
$5 \mathrm{~cm}^{2}$ and $6 \mathrm{~cm}^{2}$ which are centered at $[-\pi / 20, \pi / 5,8 \mathrm{~cm}],[\pi / 20, \pi / 5,8 \mathrm{~cm}]$ and $[\pi / 10, \pi / 6,8 \mathrm{~cm}]$ (in spherical coordinates) is localized with the help of the BCMP algorithm and cortical LORETA. As the resulting values of the TPF displayed in Figure 7 (top) show, the BCMP algorithm outperforms the cortical LORETA solutions (in terms of the TPF), especially for small numbers of time samples. Moreover, running cortical LORETA on the data preprocessed by the STWV technique enhances the performance of the source localization compared to the cortical LORETA solution based on the raw data.

Influence of noise. Another interesting question is how the performance of the BCMP algorithm depends on the level of noise. Figure 7 (bottom) shows the TPF of the BCMP and cortical LORETA source localization results as a function of the SNR for a distributed source composed of 4 patches covering about $14 \mathrm{~cm}^{2}$ of cortex. The patches are located at $[\pi / 2, \pi / 8,8 \mathrm{~cm}]$, $[11 \pi / 20,0,8 \mathrm{~cm}],[16 \pi / 30, \pi / 16,8 \mathrm{~cm}]$ and $[21 \pi / 40,19 \pi / 120,8 \mathrm{~cm}]$ (in spherical coordinates) and all comprise an area of $4 \mathrm{~cm}^{2}$. Here, applying the BCMP algorithm to EEG data with SNRs greater than $-4 \mathrm{~dB}$ yields better results than using cortical LORETA. For very low SNRs, however, cortical LORETA outperforms the BCMP algorithm. In this case, a performance gain can be observed for using cortical LORETA in combination with the STWV analysis proposed in this paper, whereas there is not much difference in the source localization accuracy for high SNRs.

Two distributed sources. A main point of interest is the localization of more than one distributed source because here the capability of the STWV analysis to separate the sources comes into play. This issue shall be addressed in the 
following, where two distributed sources with statistically independent activities are combined. The first source consists of three circular-shaped patches of sizes $5 \mathrm{~cm}^{2}, 5 \mathrm{~cm}^{2}$ and $6 \mathrm{~cm}^{2}$, which are centered at $[-\pi / 20, \pi / 5,8 \mathrm{~cm}]$, $[\pi / 20, \pi / 5,8 \mathrm{~cm}]$, and $[\pi / 10, \pi / 6,8 \mathrm{~cm}]$ (in spherical coordinates), respectively, and the second source comprises four circular-shaped patches of equal area $4 \mathrm{~cm}^{2}$ located at $[\pi / 2, \pi / 8,8 \mathrm{~cm}],[11 \pi / 20,0,8 \mathrm{~cm}],[16 \pi / 30, \pi / 16,8 \mathrm{~cm}]$, and $[21 \pi / 40,19 \pi / 120,8 \mathrm{~cm}]$ (in spherical coordinates). Applying cortical LORETA to the averaged potential data, the two sources are localized simultaneously and the TPF amounts to $81 \%$ (see Figure 8 (left)). As Figure 8 (right) shows, the STWV technique correctly separates the two sources into distinct components. If cortical LORETA is run on the preprocessed data, its performance can be increased to a TPF of $86 \%$, which confirms the hypothesis that to obtain accurate results, the STWV preprocessing should be used. The best results can be achieved if the two sources are localized with the BCMP algorithm based on the STWV decomposed data, which leads to a TPF of $91 \%$ (see Figure 8 (right)).

\section{Conclusions}

In this paper, we have shown that the newly developed STWV analysis constitutes a powerful preprocessing tool for the analysis of EEG data generated by superficial sources. This could imply that deep mesial brain structures might be difficult to localize with STWV, which would potentially constitute a burden for a further application of this method in the particular field of epilepsy. Nevertheless, such a burden is most likely to disappear with the use of realistic models of the brain which include most of the mesial cere- 
bral structures. The STWV method does not only permit to separate the sources, but simultaneously extracts the source time signals prior to the actual source localization. Compared to the STF analysis, the STWV method is particularly well suited if the data is only recorded over a small number of time samples. A potential application of the STWV technique is thus the tracing of the temporal evolution of sources. Moreover, the estimation error of the leadfield matrix is reduced, which improves the perfomance of the source localization as can be seen from the cortical LORETA solutions based on the averaged potential data and the preprocessed data. To further enhance the accuracy of distributed source localization results based on a model that assumes equal strengths of source dipoles, we have proposed to employ the iterative BCMP algorithm and have demonstrated its efficiency with the help of computer simulations. These simulations are a necessary step prior to the application of the STWV analysis to actual measured signals. Indeed they provide the "ground truth" necessary for the quantification of the performance of this method. Ultimately, further studies will assess the pertinency of the STWV analysis in combination with the BCMP algorithm for spatially distributed sources along with realistic head models and will consider the application of this method to real EEG data.

\section{Acknowledgements}

This work has been partly supported by the French ANR contract 10BLAN-MULTIMODEL. 


\section{References}

[1] R. Grech, T. Cassar, J. Muscat, K. P. Camilleri, S. G. Fabri, M. Zervakis, P. Xanthopoulos, V. Sakkalis, B. Vanrumste, Review on solving the inverse problem in EEG source analysis, Journal of NeuroEngineering and Rehabilitation 5 (2008).

[2] M. De Vos, A. Vergult, L. De Lathauwer, W. De Clercq, S. Van Huffel, P. Dupont, A. Palmini, W. Van Paesschen, Canonical decomposition of ictal scalp EEG reliably detects the seizure onset zone, NeuroImage 37 (2007) 844-854.

[3] M. De Vos, L. De Lathauwer, V. Vanrumste, S. Van Huffel, W. Van Paesschen, Canonical decomposition of ictal scalp EEG and accurate source localisation: Principles and simulations study, Computational Intelligence and Neuroscience (2007) 1-10.

[4] F. Miwakeichi, E. Martinez-Montes, P. A. Valdes-Sosa, N. Nishiyama, H. Mizuhara, Y. Yamaguchi, Decomposing EEG data into space-timefrequency components using parallel factor analysis, NeuroImage 22 (2004) 1035-1045.

[5] W. Deburchgraeve, P. J. Cherian, M. De Vos, R. M. Swarte, J. H. Blok, G. H. Visser, P. Govaert, Neonatal seizure localization using PARAFAC decomposition, Clinical Neurophysiology 120 (2009) 1787-1796.

[6] M. Morup, L. K. Hansen, C. S. Herrmann, J. Parnas, S. M. Arnfred, Parallel factor analysis as an exploratory tool for wavelet transformed event-related EEG, NeuroImage 29 (2006) 938-947. 
[7] M. Weis, F. Roemer, M. Haardt, D. Jannek, P. Husar, Multi-dimensional space-time-frequency component analysis of event related EEG data using closed-form PARAFAC, in: Proc. IEEE ICASSP, Taipei, Taiwan.

[8] R. D. Pascual-Marqui, M. Esslen, K. Kochi, D. Lehmann, Functional imaging with low resolution brain electromagnetic tomography (LORETA): review, new comparisons, and new validation, Journal of Clinical Neurophysiology 30 (2002) 81-94.

[9] D. Cosandier-Rimele, J.-M. Badier, P. Chauvel, F. Wendling, A physiologically plausible spatio-temporal model for EEG signals recorded with intracerebral electrodes in human partial epilepsy, IEEE Transactions on Biomedical Engineering 54 (2007).

[10] R. D. Pascual-Marqui, C. M. Michel, D. Lehmann, Low resolution electromagnetic tomography: A new method for localizing electrical activity in the brain, Int. Journal of Psychophysiology 18 (1994) 49-65.

[11] R. D. Pascual-Marqui, Review on methods for solving the EEG inverse problem, Int. Journal of Bioelectromagnetism 1 (1999) 75-86.

[12] J. C. Mosher, R. M. Leahy, P. S. Lewis, EEG and MEG: Forward solutions for inverse methods, IEEE Transactions on Biomedical Engineering 46 (1999).

[13] P. Comon, C. Jutten (Eds.), Handbook of Blind Source Separation, Independent Component Analysis and Applications, Academic Press, Oxford UK, Burlington USA, 2010. 
[14] T. G. Kolda, B. W. Bader, Tensor decompositions and applications, SIAM review (2008).

[15] P. Comon, L. Luciani, A. L. F. De Almeida, Tensor decompositions, alternating least squares and other tales, Journal of Chemometrics 23 (2009) 393-405.

[16] R. Grave de Peralta Menendez, S. Gonzalez Andino, G. Lantz, C. M. Michel, T. Landis, Noninvasive localization of electromagnetic epileptic activity. i. method descriptions and simulations, Brain Tomography 14 (2001).

[17] S. Mallat, Z. Zhang, Matching pursuit in a time-frequency dictionary, IEEE Transactions on Signal Processing 41 (1993) 3397-3415.

[18] B. H. Jansen, V. G. Rit, Electroencephalogram and visual evoked potential generation in a mathematical model of coupled cortical columns, Biological Cybernatics 73 (1995) 357-366.

[19] F. Roemer, M. Haardt, A closed-form solution for parallel factor (PARAFAC) analysis, in: IEEE Proc. ICASSP, Las Vegas, NV.

[20] R. Bro, Multi-way analysis in the food industry: Models, algorithms and applications, Ph.D. thesis, University of Amsterdam (NL) and Royal Veterinary and Agricultural University (DK), 1998. 


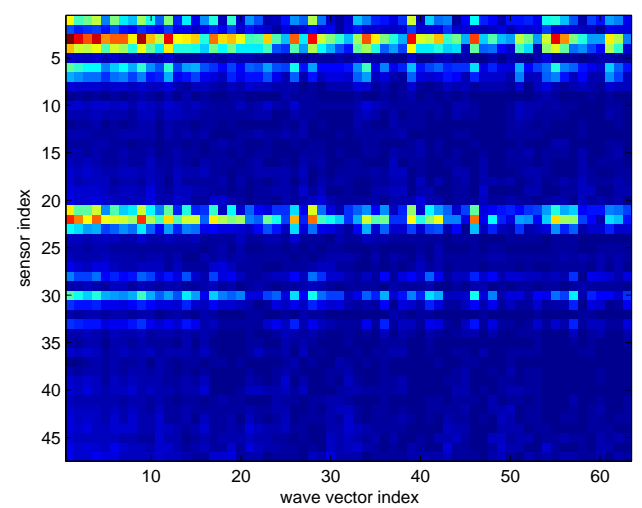

Figure 1: Space-Wave-vector data at one time instant computed from the data generated by a dipole source located at $\rho=[-\pi / 12, \pi / 5,8 \mathrm{~cm}]$ (in spherical coordinates) in the absence of noise and recorded with 64 electrodes. 

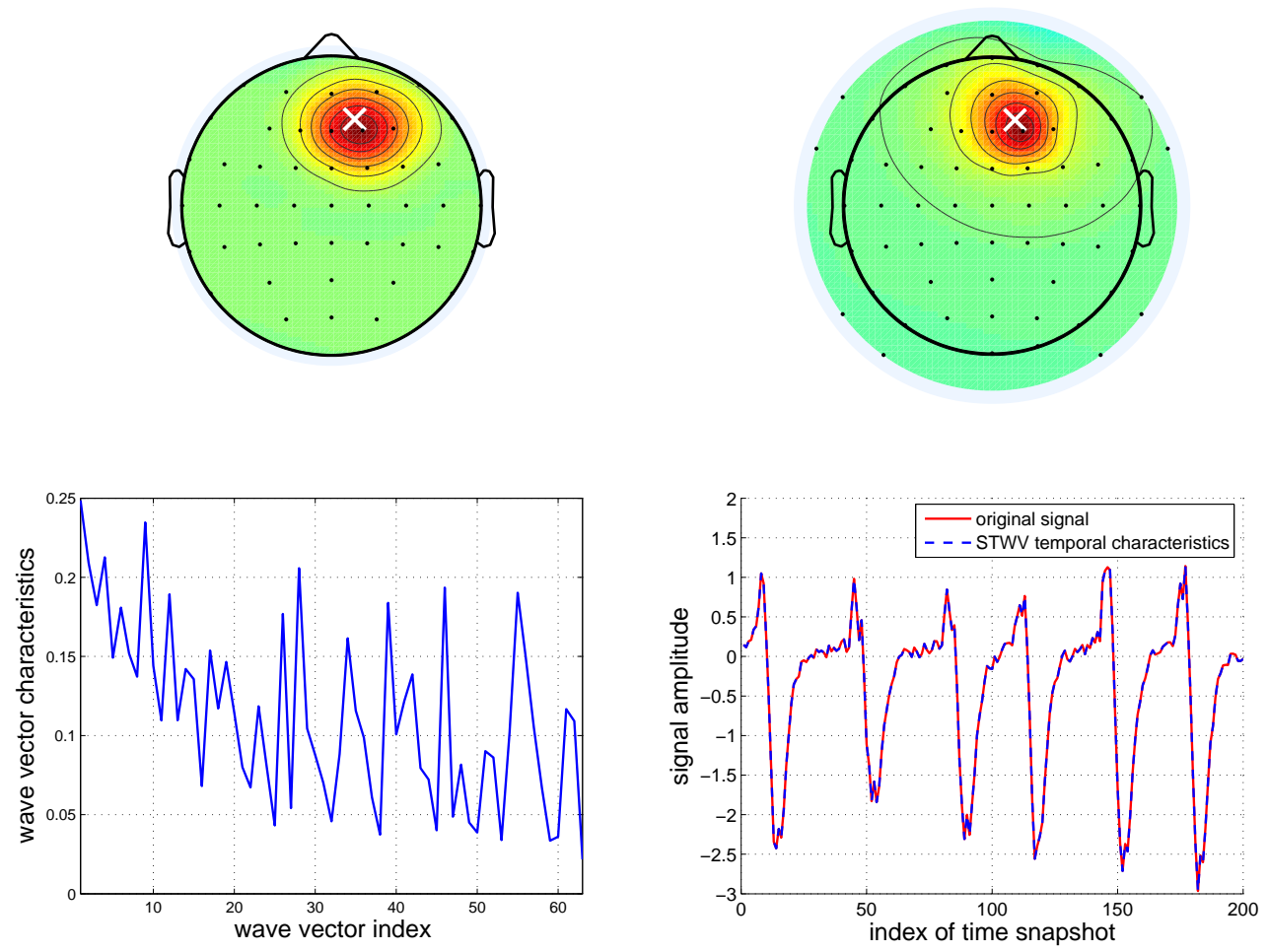

Figure 2: Space characteristics $\mathbf{a}\left(\mathbf{r}_{i}\right)$ of the STWV analysis (top left) in comparison to the original potential distribution (top right), as well as the absolute value of the wave vector characteristics $\left|\mathbf{c}\left(\mathbf{k}_{l}\right)\right|$ (bottom left) and time characteristics $\mathbf{b}\left(t_{j}\right)$ (bottom right) of the STWV tensor for EEG data generated by a dipole source located at $\boldsymbol{\rho}=[-\pi / 12, \pi / 5,8 \mathrm{~cm}]$ (in spherical coordinates) and recorded with 64 electrodes for 200 time samples in the absence of noise. The white cross in the top figures marks the dipole position. 


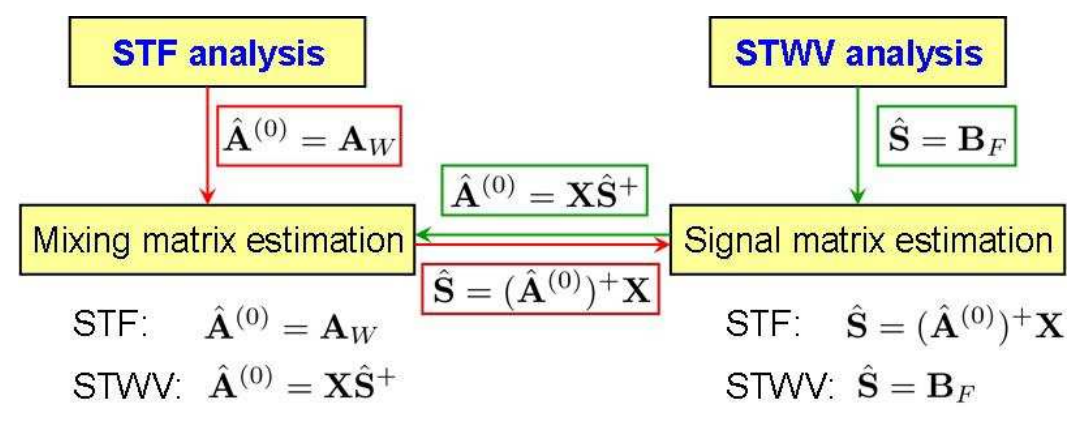

Figure 3: Leadfield and signal matrix estimation procedures for the STF and the proposed STWV analyses. 


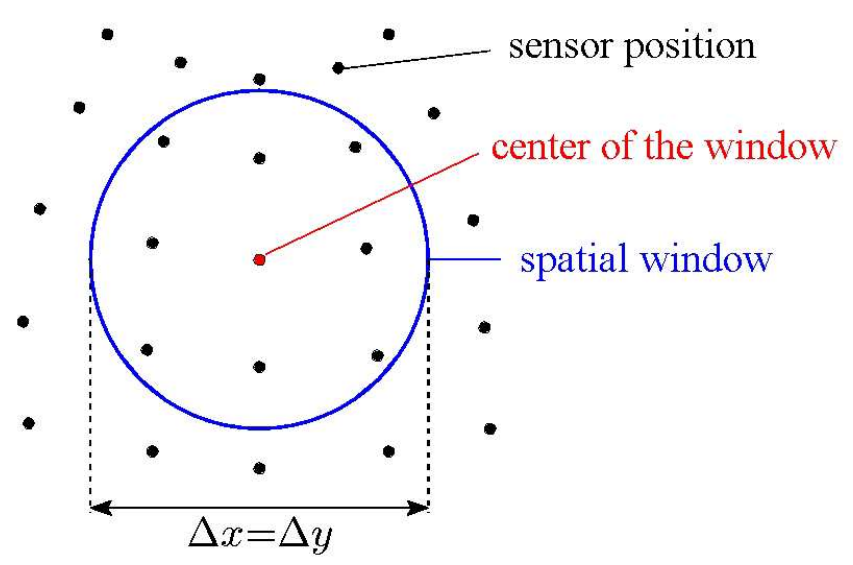

Figure 4: Selection of data in a 2-dimensional domain using a window function that is a circle. The black points mark the electrode positions, at which the electric potential is measured. The window is centered at the sensor position for which the local Fourier transform is to be computed. Data outside of the window is not considered for the transform. 

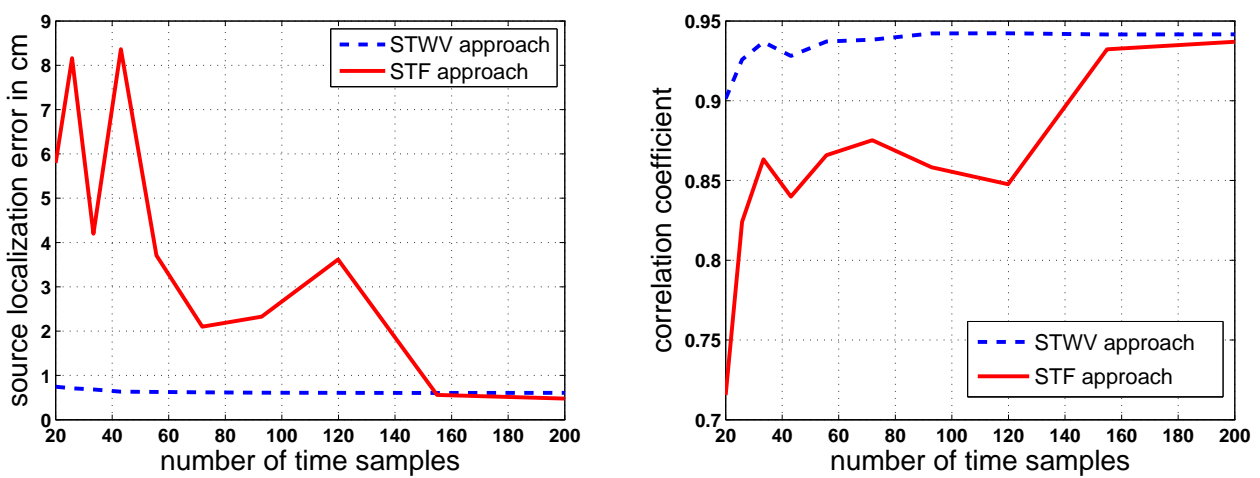

Figure 5: Source localization error (left) and correlation coefficient of original and estimated source time signals (right) for the Space-Time-Wave-Vector (STWV) and the Space-Time-Frequency (STF) analysis as a function of the number of time samples for $\mathrm{SNR}=-3 \mathrm{~dB}$ and $N_{r}=63$. 

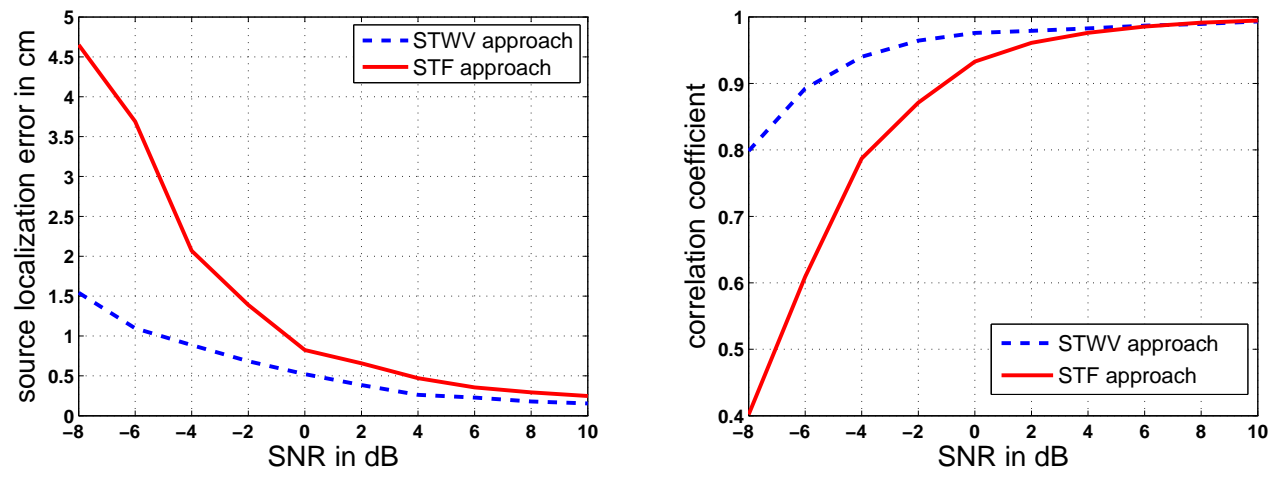

Figure 6: Source localization error (left) and correlation coefficient of original and estimated source time signals (right) for the Space-Time-Wave-Vector (STWV) and the Space-Time-Frequency (STF) analysis as a function of the SNR for $N_{t}=100$ and $N_{r}=63$. 

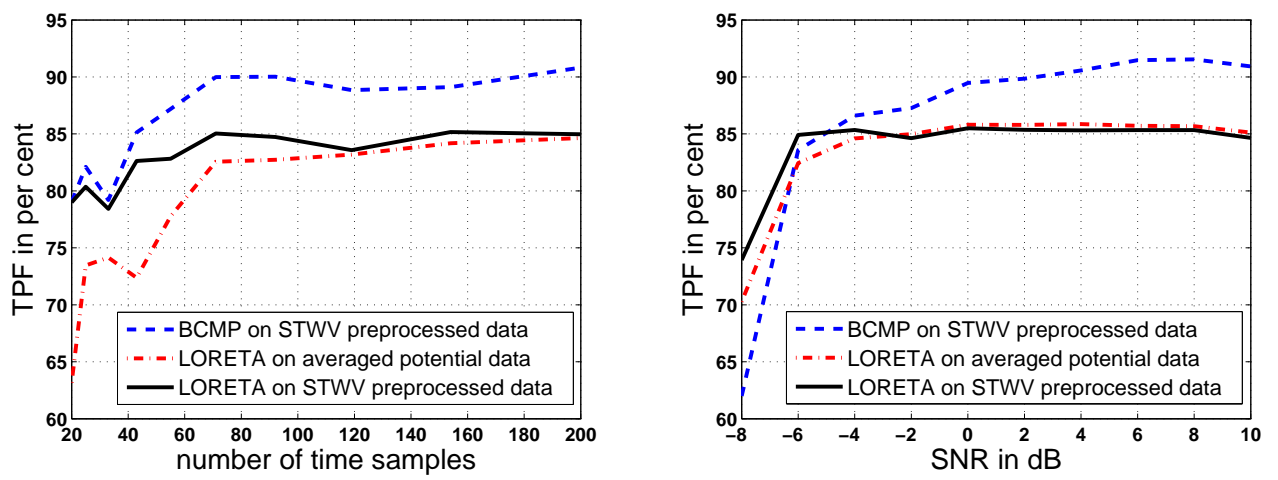

Figure 7: (Left) True positive fraction (TPF) for the Binary Coefficient Matching Pursuit (BCMP) algorithm and the two versions of cortical LORETA as a function of the number of time samples for 128 sensors and a SNR of $-3 \mathrm{~dB}$. (Right) TPF depending on the SNR for $N_{t}=100$ and $N_{r}=127$. 

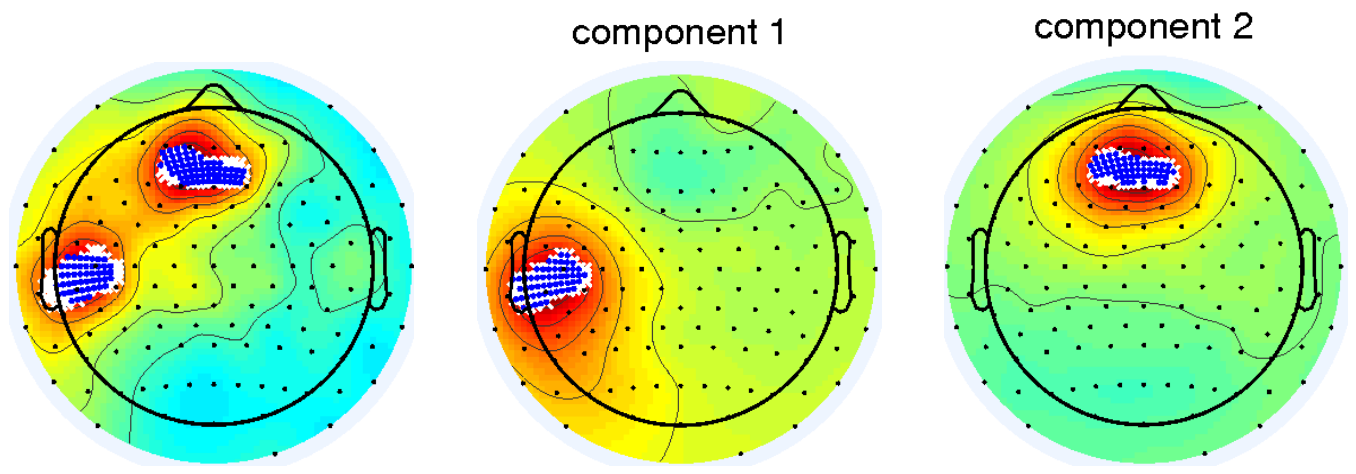

Figure 8: Topographic plots of the electric potential distribution on the surface of the scalp showing the localization results for 2 distributed sources for EEG data recorded with 128 sensors for 100 time samples and a SNR of $-3 \mathrm{~dB}$. White crosses denote the dipoles describing the original sources whereas blue points mark the estimated sources. (Left) Cortical LORETA solution based on the averaged potential distribution. (Right) Results of the Binary Coefficient Matching Pursuit (BCMP) algorithm for the 2 components extracted with the Space-Time-Wave-Vector (STWV) analysis. 